\title{
Positive Coexistence of Steady States for a Diffusive Ratio-Dependent Predator-Prey Model with an Infected Prey
}

\author{
Kwangjoong Kim and Inkyung Ahn \\ Department of Mathematics, Korea University, 2511 Sejong-Ro, Sejong 339-700, Republic of Korea \\ Correspondence should be addressed to Inkyung Ahn; ahnik@korea.ac.kr
}

Received 4 December 2014; Accepted 24 April 2015

Academic Editor: Henryk Hudzik

Copyright ( $) 2015$ K. Kim and I. Ahn. This is an open access article distributed under the Creative Commons Attribution License, which permits unrestricted use, distribution, and reproduction in any medium, provided the original work is properly cited.

\begin{abstract}
We examine a diffusive ratio-dependent predator-prey system with disease in the prey under homogeneous Dirichlet boundary conditions with a hostile environment at its boundary. We investigate the positive coexistence of three interacting species (susceptible prey, infected prey, and predator) and provide nonexistence conditions of positive solutions to the system. In addition, the global stability of the trivial and semitrivial solutions to the system is studied. Furthermore, the biological interpretation based on the result is also presented. The methods are employed from a comparison argument for the elliptic problem as well as the fixed-point theory as applied to a positive cone on a Banach space.
\end{abstract}

\section{Introduction}

This paper describes the examination of the following diffusive ratio-dependent predator-prey system with disease in the prey:

$$
\begin{aligned}
& u_{t}-\Delta u=u(a-a u-a v-v), \quad \text { in } \Omega \times(0, \infty), \\
& v_{t}-\Delta v=v\left(u-b_{2}-\frac{l w}{m w+v}\right), \\
& w_{t}-\Delta w=w\left(-b_{1}+\frac{k l v}{m w+v}\right), \\
& u(x, t)=v(x, t)=w(x, t)=0 \quad \text { on } \partial \Omega \times(0, \infty), \\
& u(x, 0)=\widetilde{u}_{0}(x) \geq 0 \quad \text { in } \Omega, \\
& v(x, 0)=\widetilde{v}_{0}(x) \geq 0, \\
& w(x, 0)=\widetilde{w}_{0}(x) \geq 0,
\end{aligned}
$$

where $\Omega \subseteq \mathbb{R}^{n}$ is a bounded region with a smooth boundary; the coefficients $a, b_{1}, b_{2}, l$, and $k$ are positive constants; the initial functions $\widetilde{u}_{0}, \widetilde{v}_{0}$, and $\widetilde{w}_{0}$ are not identically zero in $\Omega ; u$, $v$, and $w$ represent the densities of susceptible prey, infected prey, and predator, respectively. Predator $w$ preys only on infected prey $v . a$ is the intrinsic growth rate of susceptible prey $u ; b_{2}$ and $b_{1}$ are the death rates of the infected prey and predator; $m$ is known as the half saturation parameter; $l$ is the predation coefficient; $k$ represents the efficiency at which consumed prey is converted into predator births. The homogeneous Dirichlet boundary condition describes a hostile boundary environment at the boundary of the region under investigation.

Model (1) is based on the following assumptions:

(a) Prey consists of two classes: susceptible prey and infected prey.

(b) Only susceptible prey can reproduce themselves according to logistic law and infected prey, together with susceptible prey, contributes to population growth.

(c) Disease can only be spread among the prey and is not inherited.

(d) Predators only prey on infected prey.

Assumption (d) is in accordance with the fact that the infected prey is less active and can be caught more easily, or the behavior of the prey individuals is modified such that they live in parts of the habitat which are accessible to the predator (fish and aquatic snails staying close to water surface, snails staying on the top of the vegetation rather than under 
the plant cover) [1]. Also in [2], the authors indicated that wolf attacks on moose are more often successful if the moose is heavily infected by "Echinococcus granulosus." Additional background information pertaining to model (1) may be obtained from [3] and references therein.

Xiao and Chen [3] suggested the following nondimensionalized model, which is a nonspatial version of (1):

$$
\begin{aligned}
& \frac{d s}{d t}=a s[1-(s+i)]-s i, \quad s(0)>0, \\
& \frac{d i}{d t}=s i-b_{2} i-\frac{l i y}{m y+i}, \quad i(0)>0, \\
& \frac{d y}{d t}=-b_{1} y+\frac{k l i y}{m y+i}, \quad y(0)>0,
\end{aligned}
$$

where $s, i$, and $y$ denote the population density of the susceptible prey, infected prey, and predator, respectively. The initial conditions are $\mathbb{R}_{+}^{3}=\left\{(s, i, y) \in \mathbb{R}^{3}: s \geq 0, i \geq 0, y \geq\right.$ $0\}$.

Over the last three decades, predator-prey models have been studied extensively by many researchers. Among these, the ratio-dependent predator-prey models, in which the per capita predator growth rate depends on a function of the ratio of prey to predator abundance, have been proposed by Arditi and Ginzburg [4]. Since then, these models have been mathematically studied for both the spatially homogeneous [5-8] and spatially inhomogeneous cases [9-11]. The actual evidence and justification for the models have also been studied [12-15]. For more background on the model, we refer the reader to [16].

The goal of this study is to investigate the asymptotic behavior of positive solutions for (1) and the positive solutions to the steady-state system of (1):

$$
\begin{aligned}
& -\Delta u=u(a-a u-a v-v), \quad \text { in } \Omega, \\
& -\Delta v=v\left(u-b_{2}-\frac{l w}{m w+v}\right), \\
& -\Delta w=w\left(-b_{1}+\frac{k l v}{m w+v}\right), \\
& u(x)=v(x)=w(x)=0 \quad \text { on } \partial \Omega .
\end{aligned}
$$

We say that system (3) has a positive solution $(u, v, w)$ if $u(x)>0, v(x)>0$, and $w(x)>0$ for all $x \in \Omega$. The existence of positive solutions $(u, v, w)$ to system (3) is called positive coexistence.

The paper is organized as follows. In Section 2, the result for the global stability at semitrivial solution is derived. Sufficient conditions for the existence of positive solutions to system (3) are provided in Section 3, and the biological interpretations are briefly stated in Section 4.

\section{Asymptotic Behavior of Solutions}

First it should be noted that, according to the results obtained by Pao [17], the solution of (1) is unique and continuous for all positive time in $\Omega$. Moreover, because $\widetilde{u}_{0} \not \equiv 0, \widetilde{v}_{0} \not \equiv 0$, and $\widetilde{w}_{0} \not \equiv 0$ is assumed, $u(x, t), v(x, t)$, and $w(x, t)>0$ on $\bar{\Omega}$ for all $t>0$.

In this section, the global stability of the trivial and semitrivial solutions of system (1) are examined.

Our discussion is based on the following well-known fact about an eigenvalue problem.

For $q(x) \in C^{\alpha}(\bar{\Omega}), 0<\alpha<1$, consider the following eigenvalue problem:

$$
\begin{aligned}
-\Delta \phi+q(x) \phi=\lambda \phi & \text { in } \Omega, \\
\phi=0 & \text { on } \partial \Omega .
\end{aligned}
$$

Recall that problem (4) has eigenvalues $\left\{\lambda_{n}\right\}$ and eigenfunctions $\left\{\phi_{n}\right\}$ such that $\lambda_{1} \leq \lambda_{2} \leq \lambda_{3} \leq \cdots$ and $\lim _{n \rightarrow \infty} \lambda_{n}=$ $+\infty$, where $n \geq 1$. Furthermore, the eigenfunction $\phi_{1}$ of (4) corresponding to the eigenvalue $\lambda_{1}$ is unique and positive. Throughout this paper, $\lambda_{1}(q(x))$ is denoted as the principal eigenvalue $\lambda_{1}$ of the eigenvalue problem (4) corresponding to the unique positive principal eigenfunction $\phi_{1}(x)$. In addition, $\lambda_{1}(0)$ is simply denoted as $\lambda_{1}$. It is known that $\lambda_{1}(q(x))$ is a strictly increasing function, given that $q_{1}(x) \leq$ $q_{2}(x)$ and $q_{1}(x) \neq \equiv q_{2}(x)$ imply that $\lambda_{1}\left(q_{1}(x)\right)<\lambda_{1}\left(q_{2}(x)\right)$.

Consider the single equation:

$$
\begin{aligned}
-\Delta u & =u f(x, u) \quad \text { in } \Omega, \\
u & =0 \quad \text { on } \partial \Omega,
\end{aligned}
$$

where $\Omega$ is a bounded connected domain in $\mathbb{R}^{n}$ with a smooth boundary. Assume that the function $f(x, u): \bar{\Omega} \times[0, \infty) \rightarrow$ $\mathbb{R}$ satisfies the following:

(H1) $f(x, u)$ is $C^{\alpha}$-function in $x$, where $0<\alpha<1$.

(H2) $f(x, u)$ is $C^{1}$-function in $u$ with $f_{u}(x, u)<0$ for all $(x, u) \in \bar{\Omega} \times[0, \infty)$.

(H3) $f(x, u) \leq 0$ on $(x, u) \in \bar{\Omega} \times[C, \infty)$ for some positive constant $C$.

The following theorem is a consequence of the main results of [18]. In addition, one can also refer to [17, 19].

Theorem 1. (i) The nonnegative solution $u(x)$ of (5) satisfies $u(x) \leq C$ for all $x \in \bar{\Omega}$.

(ii) If $\lambda_{1}(-f(x, 0)) \geq 0$, then (5) has no positive solutions. Moreover, the trivial solution is globally asymptotically stable.

(iii) If $\lambda_{1}(-f(x, 0))<0$, then (5) has a unique positive solution which is globally asymptotically stable. In this case, the trivial solution is unstable.

Obviously, if $a>\lambda_{1}$, then system (3) only has one semitrivial solution $\left(u_{0}, 0,0\right)$, where $u_{0}$ is the unique solution to

$$
\begin{aligned}
-\Delta u & =u(a-a u) \quad \text { in } \Omega, \\
u & =0 \quad \text { on } \partial \Omega .
\end{aligned}
$$

Now the stability of the trivial solution $(0,0,0)$ and the semitrivial solution $\left(u_{0}, 0,0\right)$ is investigated. 
Theorem 2. Let $(u(x, t), v(x, t), w(x, t))$ be a positive solution of (1):

(i) If $a \leq \lambda_{1}$, then $(u, v, w) \rightarrow(0,0,0)$ as $t \rightarrow \infty$.

(ii) If $a>\lambda_{1}, \lambda_{1}\left(b_{2}-u_{0}\right) \geq 0$, and $\lambda_{1}\left(b_{1}-k l\right) \geq 0$, then $(u, v, w) \rightarrow\left(u_{0}, 0,0\right)$ ast $\rightarrow \infty$.

Proof. We only prove (ii). Since

$$
u_{t}-\Delta u \leq u(a-a u) .
$$

According to Theorem 1(iii) and the comparison principle

$$
\lim _{t \rightarrow \infty} \sup u(x, t) \leq u_{0} .
$$

Let $\epsilon$ be sufficiently small positive constant such that

$$
\epsilon<\min \left\{\lambda_{1}\left(b_{2}-u_{0}\right), \frac{a-\lambda_{1}}{a+1}\right\} .
$$

Then there exists a $T_{\epsilon} \geq 0$ such that $u(x, t) \leq u_{0}+\epsilon$ for all $t \geq T_{\epsilon}$. Thus

$$
v_{t}-\Delta v \leq v\left(u_{0}+\epsilon-b_{2}\right) .
$$

Since $\lambda_{1}\left(b_{2}-u_{0}\right)>\epsilon$, according to Theorem 1(ii) and the comparison principle, it follows that $v(x, t) \rightarrow 0$ uniformly as $t \rightarrow \infty$. Since $\lambda_{1}\left(b_{1}-k l\right)>0$, it follows that $w(x, t) \rightarrow 0$ uniformly as $t \rightarrow \infty$, again according to Theorem 1(ii). Thus, there exists a $T_{\epsilon}^{\prime} \geq 0$ such that $v(x, t) \leq \epsilon$, for all $t \geq T_{\epsilon}^{\prime}$. Thus, the following is obtained:

$$
u_{t}-\Delta u \geq u(a-a u-(a+1) \epsilon) .
$$

Since $\epsilon<\left(a-\lambda_{1}\right) /(a+1)$, Theorem 1(iii) and the comparison principle yield

$$
\lim _{t \rightarrow \infty} \inf u(x, t) \geq u_{0, \varepsilon}
$$

where $u_{0, \epsilon}$ is the positive unique solution of $-\Delta u=u(a-a u-$ $(a+1) \epsilon)$ under a homogeneous Dirichlet boundary condition. Thus, by using the continuity for $\epsilon \rightarrow 0$, it is concluded that $u(x, t) \rightarrow u_{0}$ as $t \rightarrow \infty$.

\section{Positive Steady-State Solutions}

In this section, the sufficient conditions for the existence of positive solutions of (3) are given by using fixed point index theory.

A fixed point index theorem is provided to calculate the index of certain operators for semitrivial solutions of the system.

Let $E$ be a real Banach space and $W \subset E$ a closed convex set. $W$ becomes a total wedge if $\alpha W \subset W$ for all $\alpha \geq 0$ and $\overline{W-W}=E$. A wedge is said to be a cone if $W \cap(-W)=\{0\}$. For $y \in W$, define $W_{y}=\{x \in E: y+\gamma x \in W$ for some $\gamma>0\}$ and $S_{y}=\left\{x \in \bar{W}_{y}:-x \in \bar{W}_{y}\right\}$. Then $\bar{W}_{y}$ is a wedge containing $W, y$, and $-y$, whereas $S_{y}$ is a closed subspace of $E$ containing $y$. Let $T$ be a compact linear operator on $E$ which satisfies $T\left(\bar{W}_{y}\right) \subset \bar{W}_{y}$. We say that $T$ has property $\alpha$ on $\bar{W}_{y}$ if there is a $t \in(0,1)$ and a $w \in \bar{W}_{y} \backslash S_{y}$ such that $w-t T w \in S_{y}$. Let $F: W \rightarrow W$ be a compact operator with a fixed point $y \in W$ and $F$ Fréchet differentiable at $y$. Let $L=F^{\prime}(y)$ be the Fréchet derivative of $F$ at $y$, in which case $L$ maps $\bar{W}_{y}$ into itself. For an open subset $U \subset W$, define index $_{W}(F, U)=\operatorname{index}(F, U, W)=\operatorname{deg}_{W}(I-F, U, 0)$, where $I$ is the identity map. If $y$ is an isolated fixed point of $F$, then the fixed point index of $F$ at $y$ in $W$ is defined by $\operatorname{index}_{W}(F, y)=\operatorname{index}(F, y, W)=\operatorname{index}(F, U(y), W)$, where $U(y)$ is a small open neighborhood of $y$ in $W$.

The following theorem can be obtained from the results of $[18,20,21]$.

Theorem 3. Assume that $I-L$ is invertible on $\bar{W}_{y}$ :

(i) If $L$ has property $\alpha$ on $\bar{W}_{y}$, then index ${ }_{W}(F, y)=0$.

(ii) If $L$ does not have property $\alpha$ on $\bar{W}_{y}$, then index $_{W}(F, y)=(-1)^{\sigma}$, where $\sigma$ is the sum of multiplicities of all the eigenvalues of $L$ which are greater than 1 .

Lemma 4. If $l k>b_{1}$, then any nonnegative solution $(u, v, w)$ of system (3) has an a priori bound

$$
\begin{gathered}
u(x) \leq Q_{1}, \\
v(x) \leq Q_{2}, \\
w(x) \leq Q_{3},
\end{gathered}
$$

where $Q_{1}=1, Q_{2}=\left(a+b_{2}\right) / b_{2}(1+a), Q_{3}=\left(\left(a+b_{2}\right) / b_{2}(1+\right.$ a)m) $\left(\left(k l / b_{1}\right)-1\right)$.

Proof. Consider the following elliptic problem:

$$
\begin{aligned}
-\Delta U & =U(a-a U) \quad \text { in } \Omega, \\
U & =0 \text { on } \partial \Omega .
\end{aligned}
$$

Let $(u, v, w)$ be a positive solution of (3). Then the comparison property of eigenvalues yields $0=\lambda_{1}(-a+a u+a v+v)>$ $\lambda_{1}(-a)$; hence the unique positive solution $U^{*}\left(\equiv u_{0}\right)$ to (14) exists. It is easy to show that 1 is an upper solution of (14). It follows that $U^{*} \leq 1$ in $\bar{\Omega}$. Since $(u, v, w)$ is a positive solution of (3), $\Delta u+u(a-a u-a v-v)=0$ holds in $\Omega$. Hence $\Delta u+$ $u(a-a u)>0$ is satisfied for $u>0$ and $u$ is a positive lower solution of (14); therefore, $u \leq U^{*} \leq 1$ in $\bar{\Omega}$.

Next, multiplying the second equation in (3) by $(a+1)$, and adding it to the first equation in (3), we obtain

$$
\begin{aligned}
-\Delta(u+(a+1) v) & \leq a(1-u) u-b_{2}(1+a) v \\
& \leq\left(a+b_{2}\right)-b_{2}(u+(1+a) v) .
\end{aligned}
$$

Now, take $\theta=u+(1+a) v$ and consider the equation

$$
\begin{aligned}
-\Delta \theta & =\left(a+b_{2}\right)-b_{2} \theta, \quad x \in \Omega, \\
\theta & =0, \quad x \in \partial \Omega,
\end{aligned}
$$

Then, $(1+a) v(x) \leq \theta(x) \leq\left(a+b_{2}\right) / b_{2}$, and so we obtain $v(x) \leq\left(a+b_{2}\right) / b_{2}(1+a):=Q_{2}$. 
Finally, consider the following elliptic boundary value problem:

$$
\begin{aligned}
-\Delta Z & =Z\left(\frac{Q_{2}\left(k l-b_{1}\right)-b_{1} m Z}{Q_{2}+m Z}\right) \quad \text { in } \Omega, \\
Z & =0 \quad \text { on } \partial \Omega .
\end{aligned}
$$

For a positive solution $(u, v, w)$ of (3), $0=\lambda_{1}\left(b_{1}-(k l v /(m w+\right.$ $v)))>\lambda_{1}\left(b_{1}-k l\right)$ holds according to the comparison property of eigenvalues. Hence, there exists a unique positive solution $Z^{*}$ of (17), which is less than or equal to $Q_{2} / m\left(\left(k l / b_{1}\right)-1\right)$. Moreover, $w$ becomes a positive lower solution of (17) since

$$
\begin{aligned}
0 & =\Delta w+w\left(\frac{k l v}{m w+v}-b_{1}\right) \\
& <\Delta w+w\left(\frac{k l Q_{2}}{m w+Q_{2}}-b_{1}\right) \\
& =\Delta w+w\left(\frac{Q_{2}\left(k l-b_{1}\right)-b_{1} m w}{m w+Q_{2}}\right) ;
\end{aligned}
$$

therefore $w \leq Z^{*} \leq Q_{2} / m\left(\left(k l / b_{1}\right)-1\right)$ in $\bar{\Omega}$.

Remark 5. Note that if $k l \leq b_{1}$, then $w(x) \leq 0$. Thus one cannot expect a coexistence of predator $w$ under the assumption $k l \leq b_{1}$.

Note that the given growth rates in (3) are not defined at $(u, v, w)=(0,0,0)$. To overcome this, the approach of Kuang and Beretta is adopted [8]. More precisely, since

$$
\lim _{(v, w) \rightarrow(0,0)} \frac{v w}{m w+v}=0
$$

the domain of $v w /(m w+v)$ may be extended to $\{(u, v, w)$ : $u \geq 0, v \geq 0, w \geq 0\}$ so that $(0,0,0)$ becomes a trivial solution of (3). paper.

The following notation will be used throughout this

Notation 1. (i) Consider $C_{D}(\bar{\Omega}):=\{\phi \in C(\bar{\Omega}): \phi=$ 0 on $\partial \Omega\}$.

(ii) Consider $X:=C_{D}(\bar{\Omega}) \oplus C_{D}(\bar{\Omega}) \oplus C_{D}(\bar{\Omega})$. of $X$.

(iii) $W:=C_{D}(\bar{\Omega})^{+} \oplus C_{D}(\bar{\Omega})^{+} \oplus C_{D}(\bar{\Omega})^{+}$is the positive cone

(iv) Consider $Q:=\max \left\{1,\left(a+b_{2}\right) / b_{2}(1+a),\left(a+b_{2}\right) / b_{2}(1+\right.$ a) $\left.m\left(\left(k l / b_{1}\right)-1\right)\right\}+1$.

(v) Consider $N(t):=\{z \in C(\bar{\Omega}): z=0$ on $\partial \Omega$ and $0 \leq z \leq t$ on $\bar{\Omega}\}$.

(vi) Consider $D_{\mathrm{Q}}:=N(\mathrm{Q}) \oplus N(\mathrm{Q}) \oplus N(\mathrm{Q})$.
For $\theta_{1}, \theta_{2}, \theta_{3} \in[0,1]$, the positive and compact operator $\mathscr{A}_{\theta_{1}, \theta_{2}, \theta_{3}}: \bar{D}_{\mathrm{Q}} \rightarrow X$ is defined by

$$
\begin{aligned}
& \mathscr{A}_{\theta_{1}, \theta_{2}, \theta_{3}}(u, v, w) \\
& =(-\Delta+P)^{-1}\left(\begin{array}{c}
\theta_{1} u(a-a u-a v-v)+P u \\
\theta_{2} v\left(u-b_{2}-\frac{l w}{m w+v}\right)+P v \\
\theta_{3} w\left(-b_{1}+\frac{k l v}{m w+v}\right)+P w
\end{array}\right),
\end{aligned}
$$

where $P$ is taken sufficiently large such that $3 a+(a+1) Q+2+$ $b_{2}+2 l / m+b_{1}+2 k l<P$. Since each coordinate of $\mathscr{A}_{\theta_{1}, \theta_{2}, \theta_{3}}$ is a positive and compact operator by the definition at $(u, v, w)=$ $(0,0,0), \mathscr{A}_{\theta_{1}, \theta_{2}, \theta_{3}}$ is also a positive and compact operator. Thus (3) has a positive solution if and only if $\mathscr{A}=\mathscr{A}_{1,1,1}$ has a positive fixed point.

The following lemma can be represented similarly to the proof of Lemma 4.6 in [22] if a homotopy is defined as above.

Lemma 6. Consider index $\left(\mathscr{A}, D_{\mathrm{Q}}\right)=1$, where $D_{\mathrm{Q}}$ is defined in Notation 1.

Next, the index value of $\mathscr{A}$ is calculated at $(0,0,0)$. Since the Fréchet derivative of $\mathscr{A}$ is not defined at $(u, v, w)=$ $(0,0,0)$, the difficulty is overcome by using an $\varepsilon$ perturbation $\mathscr{A}_{\varepsilon}$ of $\mathscr{A}$. More precisely, define index $(\mathscr{A},(0,0,0))=$ $\lim _{\varepsilon \rightarrow 0} \operatorname{index}\left(\mathscr{A}_{\mathcal{\varepsilon}},(0,0,0)\right)$, where

$$
\mathscr{A}_{\varepsilon}: \bar{D}_{\mathrm{Q}} \rightarrow X \text { is defined as }
$$

$$
=(-\Delta+P)^{-1}\left(\begin{array}{c}
u(a-a u-a v-v)+P u \\
v\left(u-b_{2}-\frac{l w}{m w+v+\epsilon}\right)+P v \\
w\left(-b_{1}+\frac{k l v}{m w+v+\epsilon}\right)+P w
\end{array}\right) .
$$

Note that $\lambda_{1}<a$ gives a well-definedness of index $\left(\mathscr{A}_{\varepsilon},(0,0,0)\right)$ since this index value does not depend on the particular choice of $\varepsilon$ perturbation.

Lemma 7. If $a>\lambda_{1}$, index $(\mathscr{A},(0,0,0))=0$.

Proof. If it is shown that index $\left(\mathscr{A}_{\varepsilon},(0,0,0)\right)=0$, which is independent of $\varepsilon$, then the proof will be completed by the preceding argument of this lemma. Note that $\bar{W}_{(0,0,0)}=W$ and $S_{(0,0,0)}=\{(0,0,0)\}$. Define

$$
\begin{aligned}
\mathscr{L}_{0} & :=\mathscr{A}_{\varepsilon}^{\prime}(0,0,0) \\
& =(-\Delta+P)^{-1}\left(\begin{array}{ccc}
a+P & 0 & 0 \\
0 & -b_{2}+P & 0 \\
0 & 0 & -b_{1}+P
\end{array}\right) .
\end{aligned}
$$

Then, similar to Lemma 3.3 [11], it can be shown that (i) $\mathscr{I}$ $\mathscr{L}_{0}$ is invertible on $\bar{W}_{(0,0,0)}$ and (ii) $\mathscr{L}_{0}$ has property $\alpha$. Hence, index $\left(\mathscr{A}_{\mathcal{E}},(0,0,0)\right)=0$ according to Theorem $3(\mathrm{i})$. 
Next, indices are computed for the semitrivial solutions to model (3).

Lemma 8. Assume that $a>\lambda_{1}$. If $\lambda_{1}\left(b_{2}-u_{0}\right)<0$, then index $\left(\mathscr{A},\left(u_{0}, 0,0\right)\right)=0$.

Proof. First we observe that $\bar{W}_{\left(u_{0}, 0,0\right)}=C_{D}(\bar{\Omega}) \oplus C_{D}(\bar{\Omega})^{+} \oplus$ $C_{D}(\bar{\Omega})^{+}$and $S_{\left(u_{0}, 0,0\right)}=C_{D}(\bar{\Omega}) \oplus\{0\} \oplus\{0\} . \mathscr{L}_{u_{0}}$ is denoted by

$$
\begin{aligned}
& \mathscr{A}_{\epsilon}^{\prime}\left(u_{0}, 0,0\right)=(-\Delta+P)^{-1} \\
& \qquad\left(\begin{array}{ccc}
a-2 a u_{0}+P & -(a+1) u_{0} & 0 \\
0 & u_{0}-b_{2}+P & 0 \\
0 & 0 & -b_{1}+P
\end{array}\right) .
\end{aligned}
$$

First, let $\mathscr{L}_{u_{0}}\left(\phi_{1}, \phi_{2}, \phi_{3}\right)^{T}=\left(\phi_{1}, \phi_{2}, \phi_{3}\right)^{T} \in \bar{W}_{\left(u_{0}, 0,0\right)}$. For $\phi_{3}>0$, we have $\left(\Delta-b_{1}\right) \phi_{3}=0$. However, since $\lambda_{1}\left(b_{1}\right)=$ $\lambda_{1}+b_{1}>0$, which is always true, we have $\phi_{3}=0$. Similarly, $\phi_{2}=0$ can be obtained since $\lambda_{1}\left(b_{2}-u_{0}\right)>0$. Thus, $\phi_{1}$ satisfies $\left(\Delta+a-2 a u_{0}\right) \phi_{1}=0$. Since $u_{0}$ is the unique solution of (14), $\lambda_{1}\left(-a+2 a u_{0}\right)>\lambda_{1}\left(-a+a u_{0}\right)=0$. Hence, $\phi_{1}=0$ and $\mathscr{I}-\mathscr{L}_{u_{0}}$ is invertible on $\bar{W}_{\left(u_{0}, 0,0\right)}$.

Next, since $\lambda_{1}\left(b_{2}-u_{0}\right)<0$ holds, the spectral radius of $(-\Delta+P)^{-1}\left(b_{2}-u_{0}\right) \mathscr{I}$, say $\chi$, is greater than 1 . Thus, the KreinRutman theorem gives that an eigenvalue of $(-\Delta+P)^{-1}\left(b_{2}-\right.$ $\left.u_{0}\right) \mathscr{I}$ is $\chi$ and the corresponding eigenfunction $\psi$ exists in $C_{D}(\bar{\Omega})^{+}$. If we consider $\phi \in C_{D}(\bar{\Omega})$, then $(\phi, \psi, 0) \in \bar{W}_{\left(u_{0}, 0,0\right)} \backslash$ $S_{\left(u_{0}, 0,0\right)}$ and $(\phi, \psi, 0)^{T}-(1 / \chi) \mathscr{L}_{u_{0}}(\phi, \psi, 0)^{T} \in S_{\left(u_{0}, 0,0\right)}$. This shows that $\mathscr{L}_{u_{0}}$ has property $\alpha$. Hence index $\left(\mathscr{A}_{\epsilon},\left(u_{0}, 0,0\right)\right)=$ 0 according to Theorem 3(i). Therefore, it is concluded that $\operatorname{index}\left(\mathscr{A},\left(u_{0}, 0,0\right)\right)=\lim _{\epsilon \rightarrow 0} \operatorname{index}\left(\mathscr{A}_{\epsilon},\left(u_{0}, 0,0\right)\right)=0$.

The following notation is used in the next lemma for the computation of the index of semitrivial solutions when exactly one of the species is absent.

Notation 2. For a sufficiently small $\delta$,

(i) $N_{0}(t):=\left\{z \in C(\bar{\Omega}): z=0\right.$ on $\partial \Omega$ and $t_{*}<z \leq$ $t$ on $\bar{\Omega}\}$,

(ii) $D_{3}(\delta):=N_{0}(Q) \oplus N(Q) \oplus N(\delta)$.

Here, $t_{*}$ can be taken such that $(0,0,0) \notin \bar{D}_{3}(\delta)$, but $\left(u_{0}, 0,0\right) \in \overline{D_{3}}(\delta)$.

Note that it is not necessary to consider $D_{1}(\delta)$ and $D_{2}(\delta)$ since there is no positive semitrivial solution of the forms $(0, v, w)$ and $(u, 0, w)$ for $v, w>0$ according to the assumptions of model (3). In the case of $u \equiv 0$, it is easy to see that $v \equiv 0$ and $w \equiv 0$ by a strong maximum principle. Also if $v \equiv 0$, then $w \equiv 0$, because predators only prey on infected prey according to our assumption. Thus, system (3) cannot be maintained when the prey $u$ becomes extinct from a biological point of view. Hence, within the category that ensures the subsistence of the prey $u$, it is possible to obtain conditions that guarantee the existence of positive solutions.

Next, we calculate the index value of $\mathscr{A}$ in the slice $D_{3}(\delta)$. The desired result is obtained by using the homotopy invariance of the index for another positive and compact operator $\widehat{\mathscr{A}}_{\theta_{1}, \theta_{2}, \theta_{3}}: \bar{D}_{\mathrm{Q}} \rightarrow X$, which is defined by

$$
\begin{aligned}
& \widehat{\mathscr{A}}_{\theta_{1}, \theta_{2}, \theta_{3}}(u, v, w)=(-\Delta+P)^{-1} \\
& \qquad\left(\begin{array}{c}
u\left(a-a u-\theta_{1}(a+1) v\right)+P u \\
v\left[\theta_{2}\left(u-\frac{l w}{m w+v}\right)-b_{2}\right]+P v \\
\theta_{3} w\left(-b_{1}+\frac{k l v}{m w+v}\right)+P w
\end{array}\right),
\end{aligned}
$$

for $\theta_{1}, \theta_{2}, \theta_{3} \in[0,1]$.

Lemma 9. Assume that

$$
\begin{aligned}
a & >\lambda_{1}, \\
k l-b_{1} & >\lambda_{1} .
\end{aligned}
$$

If $\lambda_{1}\left(b_{2}-u_{0}\right)<0$, then index $\left(\mathscr{A}, D_{3}(\delta)\right)=0$.

Proof. Note that if $\widehat{\mathscr{A}}_{\theta_{1}, \theta_{2}, 1}$ has no fixed points in $\partial D_{3}(\delta)$, then

$$
\begin{aligned}
\operatorname{index}\left(\mathscr{A}, D_{3}(\delta)\right) & =\operatorname{index}\left(\widehat{\mathscr{A}}_{1,1,1}, D_{3}(\delta)\right) \\
& =\operatorname{index}\left(\widehat{\mathscr{A}}_{0,0,1}, D_{3}(\delta)\right)
\end{aligned}
$$

holds by using the homotopy invariance of the index.

First, it is shown that there are no fixed points $(u, v, w)$ of $\widehat{\mathscr{A}}_{\theta_{1}, \theta_{2}, 1}$ in $\partial D_{3}(\delta)$ for all $\theta_{1}, \theta_{2} \in[0,1]$ and a sufficiently small $\delta>0$. Suppose, for the purpose of contradiction, that there are some fixed points $\left(u_{n}, v_{n}, w_{n}\right)$ of $\widehat{\mathscr{A}}_{\theta_{1}, \theta_{2}, 1}$ in $\partial D_{3}(\delta)$ for any $\delta>0$. Then for $\left\{\theta_{1, n}\right\}$ and $\left\{\theta_{2, n}\right\} \in[0,1]$, there exist the sequences $\left\{\delta_{n}\right\}$ and fixed points $\left\{\left(u_{n}, v_{n}, w_{n}\right)\right\}$ of $\widehat{\mathscr{A}}_{\theta_{1, n}, \theta_{2, n}, 1}$ such that $\left(u_{n}, v_{n}, w_{n}\right) \in \partial D_{3}\left(\delta_{n}\right)$ and $\delta_{n} \rightarrow 0$. Here, $\left(u_{n}, v_{n}\right) \notin$ $\partial\left(N_{0}(Q) \oplus N(Q)\right)$ by a choice of $Q$. Hence $w_{n}$ is contained in $\partial N\left(\delta_{n}\right)$, such that $w_{n} \rightarrow 0$ and $w_{n} \neq \equiv$ as $\delta_{n} \rightarrow 0$.

Since $w_{n} \rightarrow 0$ as $\delta_{n} \rightarrow 0$,

$$
0=\lambda_{1}\left(b_{1}-\frac{k l v_{n}}{m w_{n}+v_{n}}\right) \longrightarrow \lambda_{1}\left(b_{1}-k l\right)<0
$$

using the comparison property of eigenvalues. This is a contradiction for large values of $n$. Thus, it is possible to use the homotopy invariance in the slice $D_{3}(\delta)$ for a sufficiently small $\delta>0$.

Now let us proceed to complete the proof. For $(u, v, w) \in$ $D_{3}(\delta), \widehat{\mathscr{A}}_{0,0,1}(u, v, w)=(u, v, w)$ gives $v \equiv 0,\|w\|<\delta$, and $u \equiv u_{0}$. Here, $v \equiv 0$ and $w \equiv 0$ follow from a choice of $\delta>0$ and a strong maximum principle, respectively. Hence, $\widehat{\mathscr{A}}_{0,0,1}$ has only one fixed point $\left(u_{0}, 0,0\right)$ in $D_{3}(\delta)$. Thus, index $\left(\widehat{\mathscr{A}}_{0,0,1}, D_{3}(\delta)\right)=\operatorname{index}\left(\widehat{\mathscr{A}}_{0,0,1},\left(u_{0}, 0,0\right)\right)$. To calculate index $\left(\widehat{\mathscr{A}}_{0,0,1},\left(u_{0}, 0,0\right)\right)$, we can use $\widehat{\mathscr{A}}_{\theta_{1}, \theta_{2}, \theta_{3}, \epsilon}$ as in the proof of Lemma 8. Hence, using the assumptions $a>\lambda_{1}$ and $k l-b_{1}>$ $\lambda_{1}$, we obtain

$$
\begin{aligned}
\operatorname{index} & \left(\widehat{\mathscr{A}}_{0,0,1},\left(u_{0}, 0,0\right)\right) \\
= & \operatorname{limindex}_{\epsilon \rightarrow 0}\left(\widehat{\mathscr{A}}_{0,0,1, \epsilon},\left(u_{0}, 0,0\right)\right)=0 ;
\end{aligned}
$$


therefore index $\left(\mathscr{A}, D_{3}(\delta)\right)=\operatorname{index}\left(\widehat{\mathscr{A}}_{1,1,1}, D_{3}(\delta)\right)=$ $\operatorname{index}\left(\widehat{\mathscr{A}}_{0,0,1}, D_{3}(\delta)\right)=0$.

Using the above lemmas, sufficient conditions are provided for the existence of positive solutions of system (3).

Theorem 10. Assume $a>\lambda_{1}$ and $k l-b_{1}>\lambda_{1}$. If $\lambda_{1}\left(b_{2}-u_{0}\right)<$ 0 , then (3) has a positive solution.

Proof. Using the additivity of the index,

$$
\begin{aligned}
& \operatorname{index}\left(\mathscr{A}, D_{\mathrm{Q}}\right)-\operatorname{index}(\mathscr{A},(0,0,0)) \\
& \quad-\operatorname{index}\left(\mathscr{A}, D_{3}(\delta)\right)=1-0-0=1 \neq 0 .
\end{aligned}
$$

Hence, there exists a positive solution in $N_{0}(Q) \oplus N_{0}(Q) \oplus$ $N_{0}(Q)$.

Before this section is concluded, the nonexistence conditions of the positive solutions of system (3) are mentioned in Remark 11.

Remark 11. (i) If $a \leq \lambda_{1}$, then (3) has a trivial solution.

(ii) Assume $a>\lambda_{1}$. If $b_{1}-k l \leq \lambda_{1}$ or $\lambda_{1}\left(b_{2}-u_{0}\right) \geq 0$, then there are no nonnegative nonzero solutions of (3).

(iii) Assume $a>\lambda_{1}$. If $b_{1}-k l \leq \lambda_{1}$ or $1-b_{2} \leq \lambda_{1}$, then there are no nonnegative nonzero solutions of (3).

\section{Biological Interpretation}

This paper examined a time-independent predator-prey system with ratio-dependent functional responses incorporating susceptible prey, infected prey, and predators under homogeneous Dirichlet boundary conditions. The existence and nonexistence of positive solutions to the system were investigated.

First, sufficient conditions were provided under which the system has positive solutions, such that all three species (susceptible prey, infected prey, and predator) are able to coexist in a region with a hostile environment at its boundary. The criterion for positive coexistence is significantly influenced by the sign of the principal eigenvalues of certain types of Schrödinger operators. The results indicated that either a large predation rate or high conversion rate together with a low predator death rate as well as a low death rate of infected prey facilitates the coexistence of the system.

Next, the nonexistence results for the positive coexistence states provided the conditions for both total extinction and the extinction of predators with the coexistence of susceptible and infected prey. We observed that total extinction can occur when a susceptible low-density prey species forces infected prey and predators to become extinct, and although the density of the susceptible prey is not low, low predatorhunting or a low conversion rate may lead to the extinction of infected prey, thereby resulting in the disappearance of a predator. Our model also indicates that the extinction of a predator can occur regardless of the density of infected prey, provided the predator has a sufficiently low maximal growth rate and a high death rate.

\section{Conflict of Interests}

The authors declare that there is no conflict of interests regarding the publication of this paper.

\section{Acknowledgments}

The authors thank the anonymous referees for careful reading and valuable comments which have helped to improve the presentation of this paper. This work was supported by a Korea University Grant.

\section{References}

[1] J. C. Holmes and W. M. Bethel, "Modification of intermediate host behavior by parasites," in Behavioral Aspects of Parasite Transmission, E. U. Canning and C. A. Wright, Eds., vol. 51 of Zoological Journal of the Linnean Society, supplement 1, pp. 123149, 1972.

[2] R. O. Peterson and R. E. Page, "Wolf density as a predictor of predation rate," Swedish Wildlife Research, Supplement, vol. 1, pp. 771-773, 1987.

[3] Y. Xiao and L. Chen, "A ratio-dependent predator-prey model with disease in the prey," Applied Mathematics and Computation, vol. 131, no. 2-3, pp. 397-414, 2002.

[4] R. Arditi and L. R. Ginzburg, "Coupling in predator-prey dynamics: ratio-dependence," Journal of Theoretical Biology, vol. 139, no. 3, pp. 311-326, 1989.

[5] S.-B. Hsu, T.-W. Hwang, and Y. Kuang, "Global analysis of the Michaelis-Menten-type ratio-dependent predator-prey system," Journal of Mathematical Biology, vol. 42, no. 6, pp. 489506, 2001.

[6] S.-B. Hsu, T.-W. Hwang, and Y. Kuang, "Rich dynamics of a ratio-dependent one-prey two-predators model," Journal of Mathematical Biology, vol. 43, no. 5, pp. 377-396, 2001.

[7] S.-B. Hsu, T.-W. Hwang, and Y. Kuang, "A ratio-dependent food chain model and its applications to biological control," Mathematical Biosciences, vol. 181, no. 1, pp. 55-83, 2003.

[8] Y. Kuang and E. Beretta, "Global qualitative analysis of a ratiodependent predator-prey system," Journal of Mathematical Biology, vol. 36, no. 4, pp. 389-406, 1998.

[9] R. S. Cantrell and C. Cosner, "On the dynamics of predator-prey models with the Beddington-DeAngelis functional response," Journal of Mathematical Analysis and Applications, vol. 257, no. 1, pp. 206-222, 2001.

[10] P. Y. H. Pang and M. X. Wang, "Qualitative analysis of a ratiodependent predator-prey system with diffusion," Proceedings of the Royal Society of Edinburgh. Section A. Mathematics, vol. 133, no. 4, pp. 919-942, 2003.

[11] K. Ryu and I. Ahn, "Positive solutions for ratio-dependent predator-prey interaction systems," Journal of Differential Equations, vol. 218, no. 1, pp. 117-135, 2005.

[12] R. Arditi, L. R. Ginzburg, and H. R. Akcakaya, "Variation in plankton densities among lakes: a case for ratio-dependent predation models," The American Naturalist, vol. 138, no. 5, pp. 1287-1296, 1991.

[13] R. Arditi and H. Saiah, "Empirical evidence of the role of heterogeneity in ratio-dependent consumption," Ecology, vol. 73 , no. 5, pp. 1544-1551, 1992. 
[14] C. Cosner, D. L. DeAngelis, J. S. Ault, and D. B. Olson, "Effects of spatial grouping on the functional response of predators," Theoretical Population Biology, vol. 56, no. 1, pp. 65-75, 1999.

[15] A. P. Gutierrez, "The physiological basis of ratio-dependent predator-prey theory: a metabolic pool model of Nicholson's blowies as an example," Ecology, vol. 73, pp. 1552-1563, 1992.

[16] R. Arditi and L. R. Ginzburg, How Species Interact-Altering the Standard View on Trophic Ecology, Oxford University Press, Oxford, UK, 2012.

[17] C. V. Pao, On Nonlinear Parabolic and Elliptic Equations, Plenum Press, New York, NY, USA, 1992.

[18] L. Li, "Coexistence theorems of steady states for predator-prey interacting systems," Transactions of the American Mathematical Society, vol. 305, no. 1, pp. 143-166, 1988.

[19] S. Cano-Casanova, "Existence and structure of the set of positive solutions of a general class of sublinear elliptic nonclassical mixed boundary value problems," Nonlinear Analysis: Theory, Methods \& Applications, vol. 49, no. 3, pp. 361-430, 2002.

[20] E. N. Dancer, "On the indices of fixed points of mappings in cones and applications," Journal of Mathematical Analysis and Applications, vol. 91, no. 1, pp. 131-151, 1983.

[21] M. Wang, Z. Y. Li, and Q. X. Ye, "Existence of positive solutions for semilinear elliptic system," in School on Qualitative Aspects and Applications of Nonlinear Evolution Equations (Trieste, 1990), pp. 256-259, World Scientific Publishing, River Edge, NJ, USA, 1991.

[22] K. Ryu and I. Ahn, "Coexistence theorem of steady states for nonlinear self-cross diffusion systems with competitive dynamics," Journal of Mathematical Analysis and Applications, vol. 283, no. 1, pp. 46-65, 2003. 


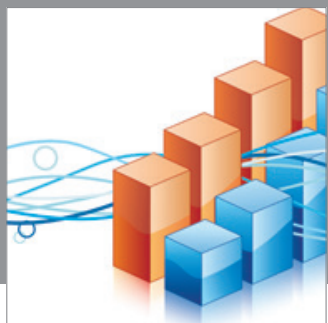

Advances in

Operations Research

mansans

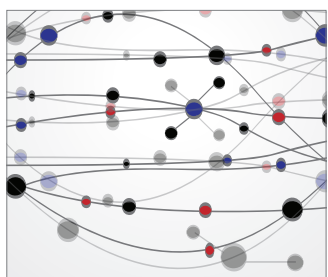

The Scientific World Journal
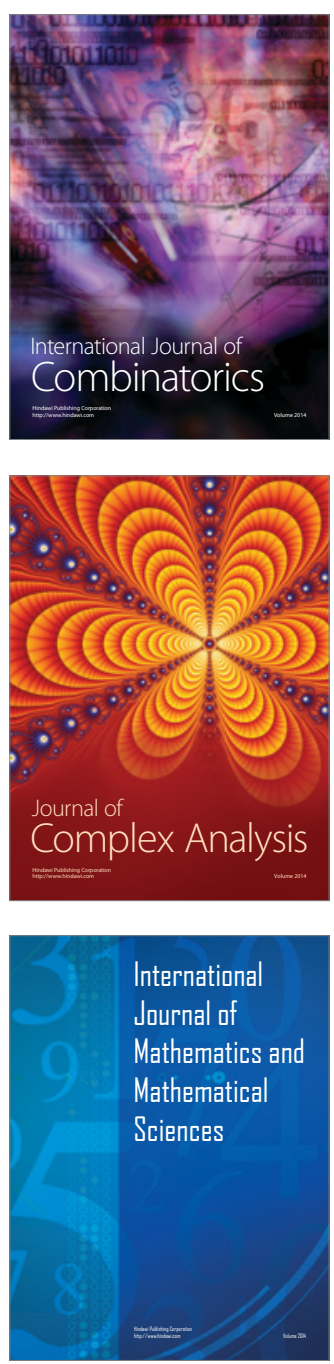
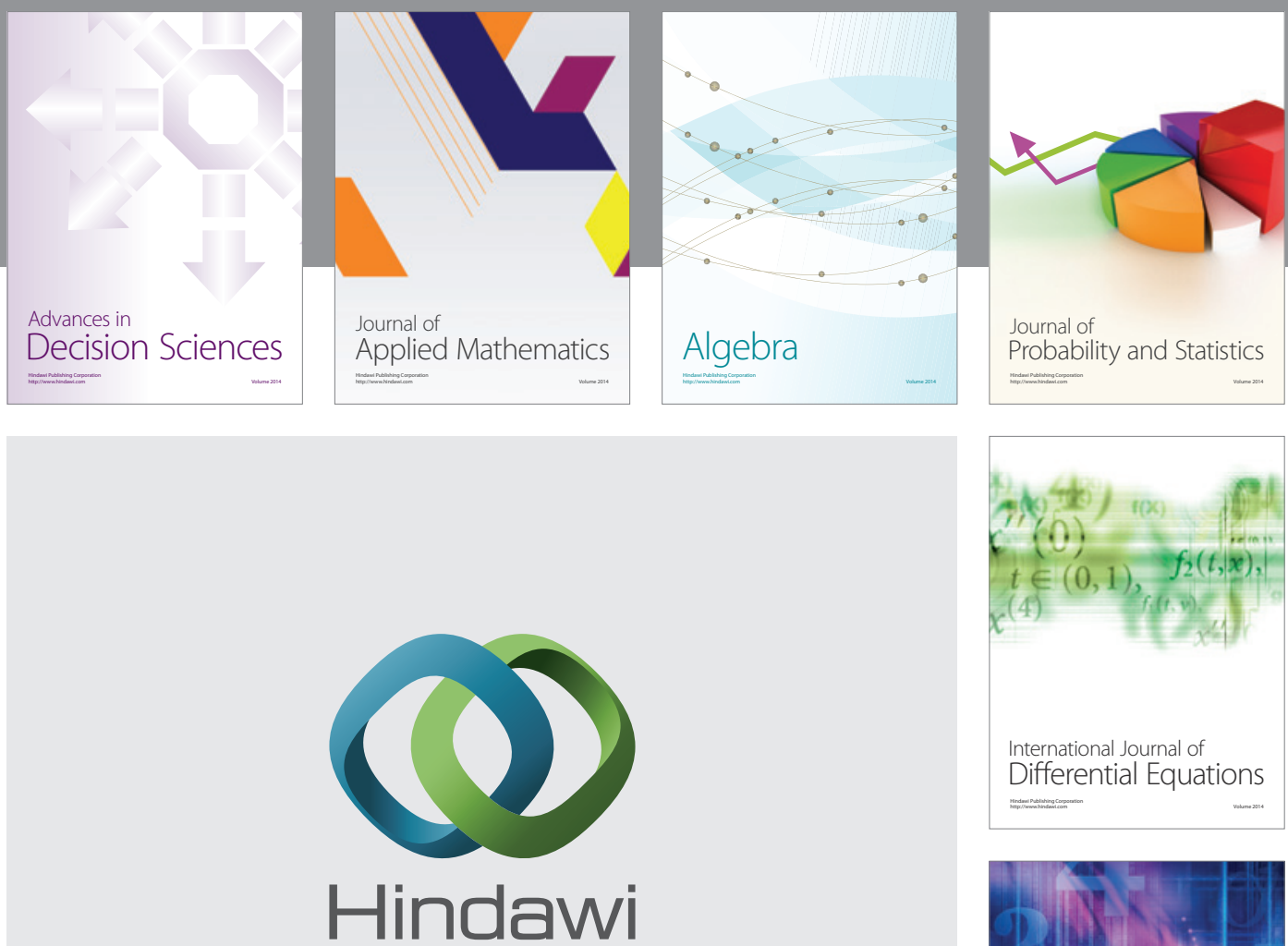

Submit your manuscripts at http://www.hindawi.com
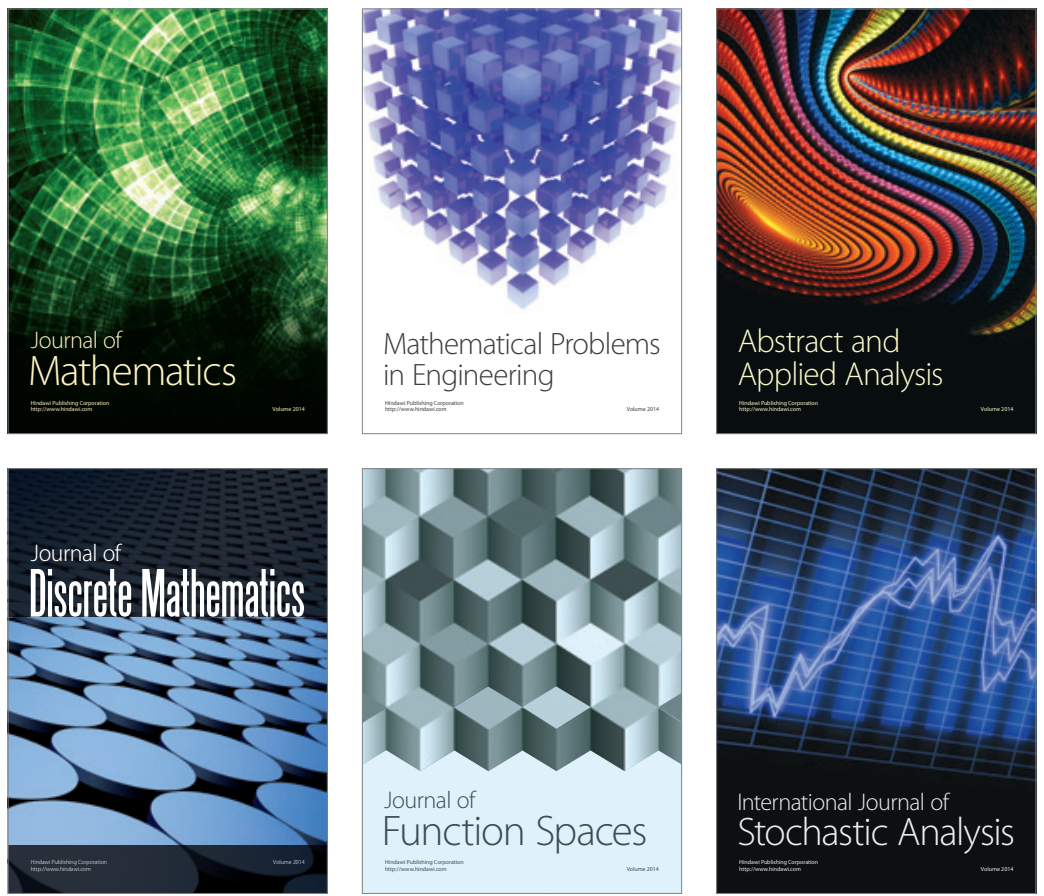

Journal of

Function Spaces

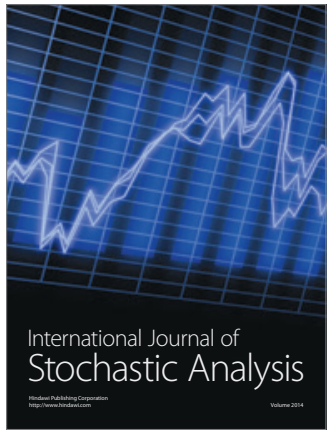

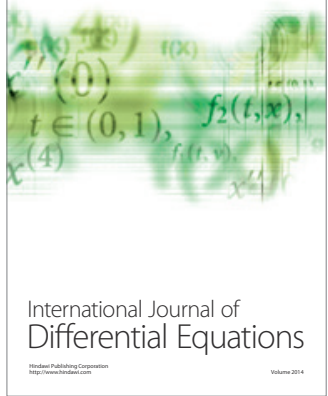
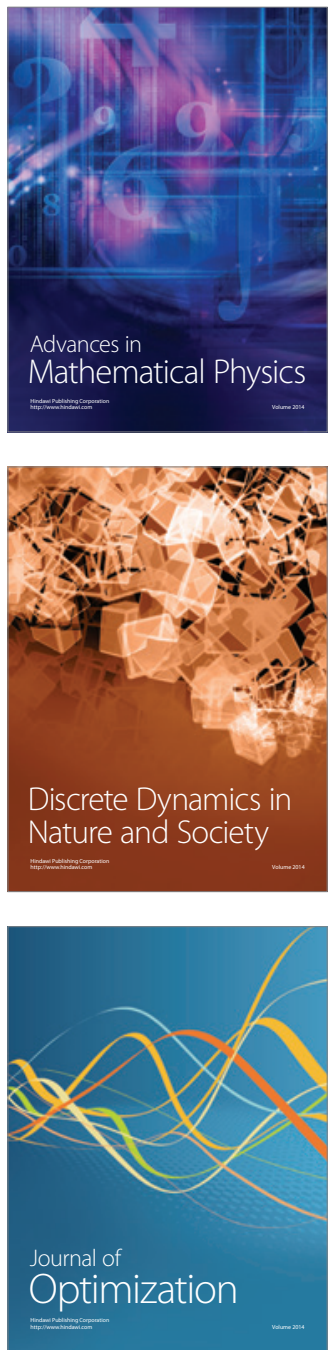